\title{
Dense Molecular Gas Tracers in the Outflow of the Starburst Galaxy NGC 253
}

\author{
Fabian Walter ${ }^{1,2,3}$, Alberto D. Bolatto ${ }^{4,5}$, Adam K. Leroy ${ }^{6}$, Sylvain Veilleux ${ }^{4}$, Steven R. Warren ${ }^{4,7}$, Jacqueline Hodge ${ }^{8}$, \\ Rebecca C. Levy ${ }^{4}$, David S. Meier ${ }^{2,9}$, Eve C. Ostriker ${ }^{10}$, Jürgen Ott ${ }^{2}$, Erik Rosolowsky ${ }^{11}$, Nick Scoville ${ }^{3}$, Axel Weiss ${ }^{12}$, \\ Laura Zschaechner ${ }^{1}$, and Martin Zwaan ${ }^{13}$ \\ ${ }^{1}$ Max Planck Institute für Astronomie, Königstuhl 17, D-69117, Heidelberg, Germany \\ ${ }^{2}$ National Radio Astronomy Observatory, P.O. Box O, 1003 Lopezville Road, Socorro, New Mexico 87801, USA \\ ${ }^{3}$ Astronomy Department, California Institute of Technology, MC105-24, Pasadena, California 91125, USA \\ ${ }^{4}$ Department of Astronomy, Laboratory for Millimeter-wave Astronomy, and Joint Space Institute, University of Maryland, College Park, Maryland 20742, USA \\ ${ }^{5}$ Visiting, Max-Planck Institute for Astronomy, Heidelberg, Germany \\ ${ }^{6}$ Department of Astronomy, Ohio State University, $100 \mathrm{~W} 18$ th Avenue, Columbus, OH 43210, USA \\ ${ }_{8}^{7}$ Cray, Inc., 380 Jackson Street, Suite 210, St. Paul, MN 55101, USA \\ ${ }^{8}$ Leiden Observatory, Niels Bohrweg 2, 2333 CA Leiden, Netherlands \\ ${ }^{9}$ New Mexico Institute of Mining \& Technology, 801 Leroy Place, Socorro, NM 87801, USA \\ ${ }^{10}$ Department of Astrophysical Sciences, Princeton University, Princeton, New Jersey 08544, USA \\ ${ }^{11}$ Department of Physics, University of Alberta, Edmonton, AB, Canada \\ ${ }^{12}$ Max-Planck-Institut für Radioastronomie, Auf dem Hügel 69, Bonn, Germany \\ ${ }^{13}$ European Southern Observatory, Karl-Schwarzschild-Strasse 2, D-85748 Garching, Germany \\ Received 2016 August 8; revised 2016 December 16; accepted 2016 December 28; published 2017 February 1
}

\begin{abstract}
We present a detailed study of a molecular outflow feature in the nearby starburst galaxy NGC 253 using ALMA. We find that this feature is clearly associated with the edge of NGC 253's prominent ionized outflow, has a projected length of $\sim 300 \mathrm{pc}$, with a width of $\sim 50 \mathrm{pc}$, and a velocity dispersion of $\sim 40 \mathrm{~km} \mathrm{~s}^{-1}$, which is consistent with an ejection from the disk about $1 \mathrm{Myr}$ ago. The kinematics of the molecular gas in this feature can be interpreted (albeit not uniquely) as accelerating at a rate of $1 \mathrm{~km} \mathrm{~s}^{-1} \mathrm{pc}^{-1}$. In this scenario, the gas is approaching an escape velocity at the last measured point. Strikingly, bright tracers of dense molecular gas $\left(\mathrm{HCN}, \mathrm{CN}, \mathrm{HCO}^{+}\right.$, $\mathrm{CS})$ are also detected in the molecular outflow: we measure an $\mathrm{HCN}(1-0) / \mathrm{CO}(1-0)$ line ratio of $\sim 1 / 10$ in the outflow, similar to that in the central starburst region of NGC 253 and other starburst galaxies. By contrast, the $\mathrm{HCN} / \mathrm{CO}$ line ratio in the NGC 253 disk is significantly lower $(\sim 1 / 30)$, similar to other nearby galaxy disks. This strongly suggests that the streamer gas originates from the starburst, and that its physical state does not change significantly over timescales of $\sim 1$ Myr during its entrainment in the outflow. Simple calculations indicate that radiation pressure is not the main mechanism for driving the outflow. The presence of such dense material in molecular outflows needs to be accounted for in simulations of galactic outflows.
\end{abstract}

Key words: galaxies: individual (NGC 253) - galaxies: ISM - galaxies: starburst - ISM: jets and outflows

\section{Introduction}

Galactic-scale winds are a ubiquitous phenomenon in both starburst galaxies and galaxies that host active galactic nuclei (e.g., Veilleux et al. 2013). They are thought to be especially important at high redshift, where strongly star-forming galaxies on the so-called galaxy "main-sequence" dominate the star formation budget, and the number density of active galactic nuclei is greater. Winds provide negative mechanical feedback and have been invoked to resolve a number of important issues in cosmology and galaxy evolution (see, e.g., Veilleux et al. 2005 for a review). Although galactic winds have been detected in galaxies out to high redshift, only in the nearby universe can they be observed panchromatically in emission to understand the mechanisms responsible for ejecting the gas. Steadily expanding observations and simulations show that winds include cold (neutral atomic and molecular) gas components, both in starburst-driven as well as AGN-driven outflows (e.g., in the case of M82: Walter et al. 2002; Engelbracht et al. 2006; Roussel et al. 2010; Leroy et al. 2015a, other galaxies: e.g., Rupke et al. 2002; Alatalo et al. 2011; Aalto et al. 2012; Meier \& Turner 2012; Rupke \& Veilleux 2013), possibly through the entrainment of ambient material. This cold gas is very difficult to measure, but likely constitutes the mass-dominant phase in galactic winds.
Mechanisms that have been proposed to drive cool winds include direct radiation forces (e.g., Murray et al. 2011), cosmic-ray pressure gradients (e.g., Uhlig et al. 2012), pressure due to supernovae-driven superbubbles (Fujita et al. 2009; Bolatto et al. 2013), and progressive entrainment into the ionized flow facilitated by Kelvin-Helmholtz instabilities (Heckman et al. 2000). Recent models suggest that the cold gas could also be emerging from condensations of the hot ionized gas phase through runaway thermal instabilities (e.g., Faucher-Giguère \& Quataert 2012; Nayakshin \& Zubovas 2012; Zubovas \& King 2012; Bustard et al. 2016; Thompson et al. 2016). The latter predictions imply a small spatial offset between the hot burst and the emergence of cold material condensing out of the wind. In most cases, observational constraints on the velocity of the outflowing gas, both neutral and ionized, indicate that the gas does not reach the escape velocity (above references), i.e., that the currently outflowing gas may be re-accreted at later cosmic times.

NGC 253 is one of the best laboratories to study starburstdriven galactic-scale winds in detail due to its proximity $(D=3.5 \mathrm{Mpc}$, Rekola et al. 2005). It is known for the galactic wind emerging from its central 200 pc (e.g., Sharp \& BlandHawthorn 2010). The wind is seen in $\mathrm{H} \alpha$ and X-ray emission (Strickland et al. 2000, 2002; Westmoquette et al. 2011), in neutral gas (Heckman et al. 2000), warm $\mathrm{H}_{2}$ (Veilleux et al. 
2009), and in $\mathrm{OH}$ emission and absorption (Turner 1985; Sturm et al. 2011). HST imaging reveals the entrained dust emission in optical broadband imaging, already suggesting that the wind also carries significant amounts of molecular gas.

ALMA cycle 0 imaging revealed the molecular wind in NGC 253 in CO(1-0) emission (Bolatto et al. 2013). This molecular wind carries enough mass to substantially shorten the current star formation episode in this galaxy, or to definitively quench star formation over a much longer period if a substantial fraction of the gas were to reach escape velocity. In the interferometer imaging, the wind breaks up into molecular filaments or "streamers" emerging from the central starburst area. The brightest of these streamers is west of the central starburst and points to the south outlining an edge of the approaching side of the approximately conical ionized outflow (Westmoquette et al. 2011); we hereafter refer to it as the (southwest) SW streamer. In Meier et al. (2015), we present a cartoon of the central starburst region of NGC 253 and discuss other tracers of the molecular gas found in the central starburst region of NGC 253, while in Leroy et al. (2015b) we focus on the properties of the giant molecular clouds and the structure of the starburst itself.

In this study, we present and discuss the properties of this brightest outflowing molecular gas streamer, as obtained from the combined cycle 0 and 1 ALMA observations together with new IRAM single-dish data. This paper is structured as follows. Section 2 summarizes the interferometric, single-dish, and Hubble Space Telescope observations, and Section 3 shows the results. In Section 3, we present a discussion of our findings, and Section 4 summarizes our conclusions. We assume a distance to NGC 253 of $D=3.5 \mathrm{Mpc}$ (Rekola et al. 2005 ), leading to a linear (projected) scale of $1^{\prime \prime}=17 \mathrm{pc}$.

\section{Observations}

\subsection{ALMA Observations}

The data presented here are based on ALMA cycle 0 and 1 observations and include IRAM $30 \mathrm{~m}$ and Mopra single dish observations to account for the missing short spacings in the interferometric ALMA imaging.

Two frequency setups were observed in ALMA band 3, one high-frequency setup (LSB: 99.8-103.7 GHz; USB: 111.8-115.7 GHz), that covers the (redshifted) $\mathrm{CO}(1-0)$ and CN lines, and one low-frequency setup (LSB: 85.6-89.6 GHz; USB: 97.4-101.4 GHz) that covers key high-density tracer molecules, such as $\mathrm{HCN}, \mathrm{HNC}$, and $\mathrm{HCO}^{+}$(see Meier et al. 2015 for a full overview of all transitions covered by the observations). Additional details about the ALMA observations and reduction can be found in Bolatto et al. (2013), Leroy et al. (2015b), and Meier et al. (2015). The ALMA band 3 interferometric observations include the following.

ALMA high-frequency setup: a seven-point mosaic targeting the $\mathrm{CO}(1-0)$ line to map the central $1 \mathrm{kpc}\left(\sim 1^{\prime}\right)$ of NGC 253 in the $\mathrm{CO}(1-0)$ transition. These observations used the following calibrators: J0038-2459 (phase), J2357-5311 (bandpass), and Uranus (amplitude). Cycle 1 observations were obtained on 2013 November 19, 2013 December 01, and (twice) on 2013 December 02. The total on-source integration time was $2.5 \mathrm{hr}$, using typically 36 antennas. These observations were complemented by a $2.6 \mathrm{hr}$, seven-pointing mosaic with the ALMA compact array (ACA) covering $\sim 2^{\prime} \times 2^{\prime}(2 \times 2 \mathrm{kpc})$ at $75 \mathrm{pc}$ resolution (cycle 1). These ACA observations used the following calibrators: J0038-2459 (phase), J2258-2758 (bandpass), and Neptune (amplitude). The ACA observations were executed on 2013 October 07, twice on 2013 November 01, three times on 2013 November 05, three times on 2013 November 06, and once on 2013 November 14. The ACA observations were obtained using nine antennas.

ALMA Low-frequency setup: a three-pointing mosaic at $90 \mathrm{GHz}$ along the NGC 253 bar (cycle 1), targeting the highdipole molecules $\mathrm{CS}(2-1), \mathrm{HCN}(1-0), \mathrm{HCO}^{+}(1-0)$, and a host of other molecules contained in the band (Meier et al. 2015). These observations used the following calibrators: J0038-2459 (phase), J2258-2758 (bandpass), and Neptune (amplitude). Observations were done on 2014 August 31, with a total of 27.5 minutes on-source using 32 antennas.

The cycle 1 data were combined with the cycle 0 data published in Bolatto et al. (2013) and Meier et al. (2015), and the combined data were used in the analysis by Leroy et al. (2015b). Because the continuum in the NGC 253 starburst is very bright, it is possible to self-calibrate on it. We applied selfcalibration to remove residual phase and flux calibration errors inherent to the data and attain a dynamic range higher than otherwise possible. Phase information using the continuum were self-calibrated first followed by amplitude self-calibration.

Natural and Briggs robust-weighted, continuum-subtracted, cubes were created at $5 \mathrm{~km} \mathrm{~s}^{-1}$ velocity resolution. All values were primary beam corrected for all quantitative analyses. As a final step, for all comparisons, we convolve the low- and highfrequency setups to a common beam size of 1!"9 (32 pc). The final robust-weighed $\mathrm{CO}(1-0)$ cube has an rms of $1.6 \mathrm{mJy}$ beam $^{-1}$ in $5 \mathrm{~km} \mathrm{~s}^{-1}$ channels and a beam size of 1 !" $6 \times 1$ !" 2 with PA of 71.5 . In velocity ranges where a signal is present, deconvolution artefacts dominate the noise (despite self-calibration and careful cleaning the data is dynamic-range limited), and the practical noise floor is closer to $6.5 \mathrm{mJy}_{\text {beam }}{ }^{-1}$. For the $\mathrm{HCN}, \mathrm{CN}, \mathrm{CS}$, and $\mathrm{HCO}^{+}$lines, the rms is $1 \mathrm{mJy}^{-1}$ beam $^{-1}$ for a $1.9 \times 1{ }^{\prime \prime} 9$ beam.

\subsection{Single Dish Observations}

In the absence of ALMA total power measurements, we have corrected the ALMA interferometric imaging for zero spacings using the following single dish telescopes.

Mopra: We use measurements obtained by the Mopra telescope to correct the ALMA $\mathrm{CO}(1-0)$ observations (highfrequency setup), as discussed in Bolatto et al. (2013), Meier et al. (2015), and Leroy et al. (2015b).

IRAM $30 \mathrm{~m}$ : We use new IRAM $30 \mathrm{~m}$ telescope measurements to correct the high-density tracer molecules (lowfrequency setup). These observations cover all relevant tracer molecules, e.g., $\mathrm{HCN}, \mathrm{HCO}^{+}$, and $\mathrm{CS}$ (project 209-14 during 2015 March 4-9). NGC 253 was above $20^{\circ}$ for $\sim 4.5 \mathrm{hr}$ each day, resulting in a total of $12.6 \mathrm{hr}$ of on-source time with exceptional observing conditions. To map a $2^{\prime} \times 2^{\prime}$ field around the center of NGC 253 with uniform sensitivity, we obtained 36 back and forth on-the-fly scan-maps with $4^{\prime \prime}$ spacing, alternating both R.A. and decl. We used the E090 HIGHDENS receiver setup spanning $81.335-89.335 \mathrm{GHz}$ in the LSB and $97.335-105.335 \mathrm{GHz}$ in the USB, allowing for simultaneous observations of high-density tracers including $\mathrm{HCO}^{+}, \mathrm{HCN}$, and CS. We observed Mars for pointing, focus, and calibration. The map has a sensitivity of $4 \mathrm{mK}$ in a $3 \mathrm{~km} \mathrm{~s}^{-1}$ channel at $89 \mathrm{GHz}$. The frequency-dependent beam size ranges from $26^{\prime \prime}$ to $29^{\prime \prime}$. 

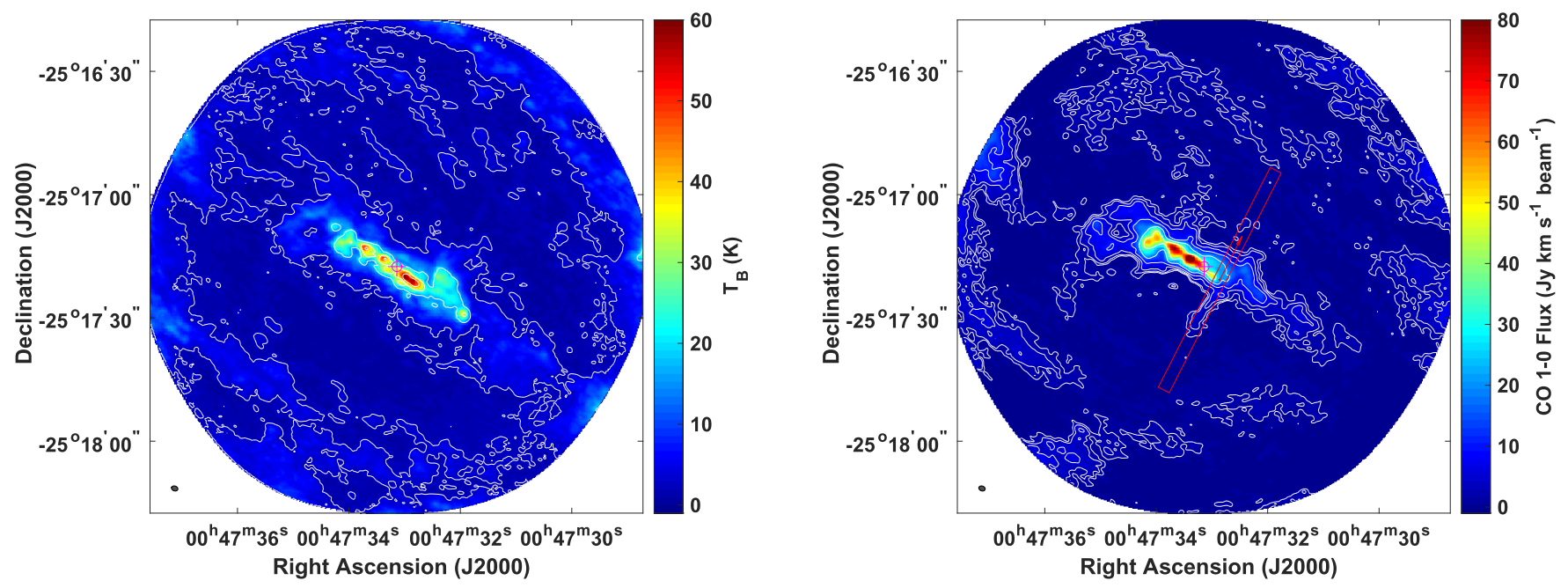

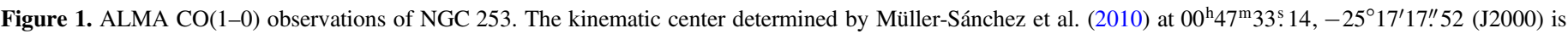

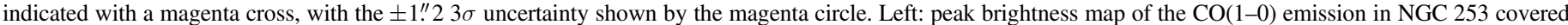

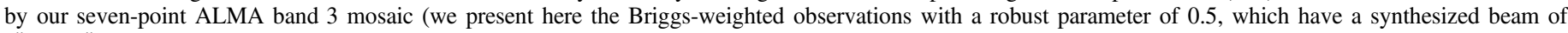

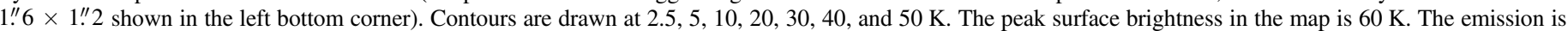

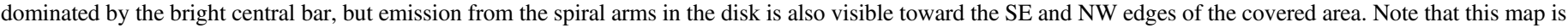

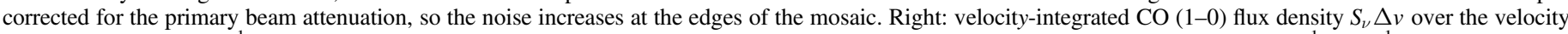

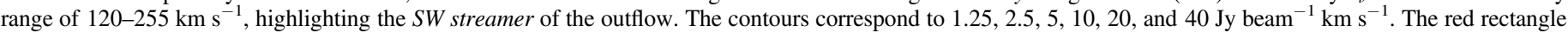

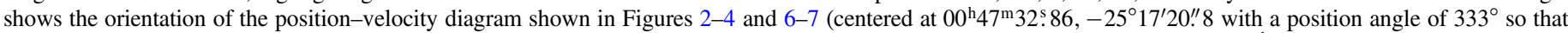

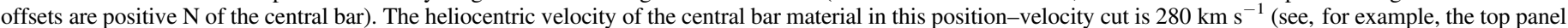
of Figure 7).

In the case of $\mathrm{CO}(1-0)$, we combined the ALMA cube with the Mopra single dish data using the feathering Miriad task immerge with a gain of $16.2 \mathrm{Jy} / \mathrm{K}$. The resulting cube was used as the model input in the CASA clean task on the ALMA-only data. We combined zero-spacings for the highdensity tracer observations from the IRAM $30 \mathrm{~m}$ telescope with the ALMA cubes using the CASA task feather. For the ALMA-IRAM $30 \mathrm{~m}$ combination, prior to feathering, we applied a gain factor of $6.15 \mathrm{Jy} / \mathrm{K}$ on the $30 \mathrm{~m}$ cubes. As in the $\mathrm{CO}(1-0)$ combination, the final cubes were created using the feathered cubes as models for the inversion of the ALMA data. This was done by including the feathered cubes as models in the ALMA data set using setjy, followed by clean using the same parameters as those used to create the original ALMA cubes. In both cases, this zero spacing correction significantly improved the negative bowls in the original (ALMA $12 \mathrm{~m}$ only) data cubes.

\subsection{Hubble Space Telescope Observations}

We compare our ALMA observations to the ionized gas outflow, traced by Hubble Space Telescope imaging of hydrogen recombination lines. We use a WFPC2 $\mathrm{H} \alpha$ image from Proposal ID 5211, first presented in Watson et al. (1996). That project observed NGC 253 in the F656N (on) and F675W (off) filters. We pair this with Paschen- $\beta$ imaging from two projects (Proposal IDs 12206 and 13730), which observed the nuclear region in the F128N (on) and F130N (off) filters. In both cases, we use the Hubble legacy archive enhanced data products, beginning with the drizzled images. For the $\mathrm{H} \alpha$, we found it necessary to combine the two visits to reject cosmicray artifacts. Then, for both sets of images, we aligned the onand off-line filters and fit a median scaling between the two in a region near but not in the nucleus. The scaling factor derived was 0.0241 and 0.95 for F675W and F130N, respectively. We used this scaling to subtract the stellar contamination from the narrow-band online image. We then converted the resulting continuum-subtracted image to have units of erg s $\mathrm{s}^{-1} \mathrm{~cm}^{-2} \mathrm{sr}^{-1}$ using the observatory supplied counts-to-flux conversion (PHOTFLAM keyword) and bandpass width (РHOTBW keyword). The values employed for $\mathrm{H} \alpha$ and Paschen- $\beta$ were $1.461 \times 10^{-16}$ and $4.278 \times 10^{-19}$ for PHOTFLAM, and 53.768 and 357.438 for PHOTBW, respectively. Finally, both images were convolved to 1.15 resolution and aligned to the ALMA astrometric grid.

\section{Results and Discussion}

\subsection{CO(1-0) Map of the Full FOV}

For reference, we show the $\mathrm{CO}(1-0)$ peak brightness map of our ALMA mosaic in Figure 1. The emission is dominated by the central bar in NGC 253 that hosts the nuclear starburst. The second panel in Figure 1 shows the $\mathrm{CO}(1-0)$ emission integrated over a velocity range that highlights the $S W$ streamer. The position and orientation of the position-velocity cut used in our subsequent analysis of the SW streamer is illustrated by the red box.

\subsection{Spectra Along the SW Streamer}

The position-velocity ( $p v$ ) diagram of the $S W$ streamer is shown in Figure 2, where the prominent outflow is highlighted with dashed red lines; these lines represent the FWHM resulting from Gaussian fitting as a function of position offset. As the orientation of this $p v$ diagram is essentially perpendicular to the major axis, we do not expect the velocities to be affected by the rotation of the disk. A different view of the same data is shown in Figure 3, which plots the $\mathrm{CO}(1-0)$ spectra along the $p v$ diagram for offsets. The spectral features identified with the streamer and the results from the Gaussian fits (central velocity, peak, and velocity width) are also displayed in Figure 3. 


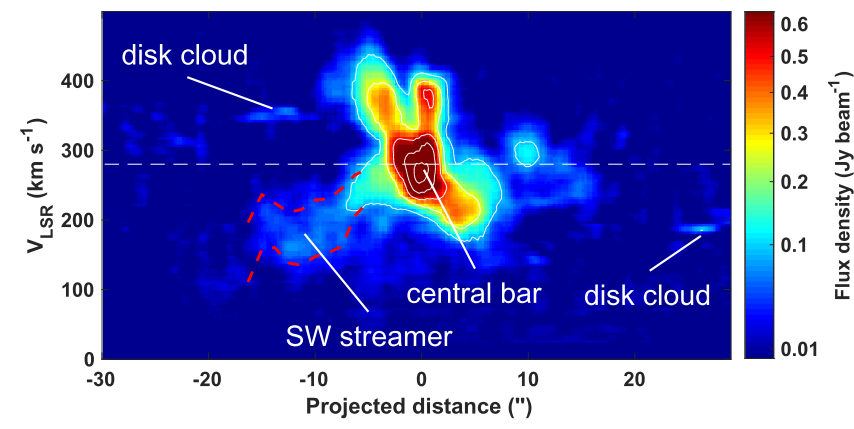

Figure 2. Position-velocity $\mathrm{CO}(1-0)$ diagram along the slit shown in Figure 1 (equivalent to a long-slit spectrum). The emission is dominated by the intense emission from the central starburst region. The narrow features at offsets of $\sim \pm 20^{\prime \prime}$ are due to molecular clouds in the galaxy's rotating disk (labeled disk cloud). The CO (1-0) outflow SW streamer (negative offsets) is also labeled. The white dashed line shows the adopted reference velocity for emission in the central bar at this location in the galaxy, of $280 \mathrm{~km} \mathrm{~s}^{-1}$. The contours indicate $\mathrm{S}_{\nu}$ of $0.09,0.25,0.49,0.81$, and $1.21 \mathrm{Jy} \mathrm{beam}^{-1}$. The color scale uses a square root stretch, and the noise in the data is $1.6 \mathrm{mJy} \mathrm{beam}^{-1}$ in emission-free velocity ranges. A typical brightness for streamer material between the dashed lines is $40-60 \mathrm{mJy}^{\text {beam }}{ }^{-1}$.

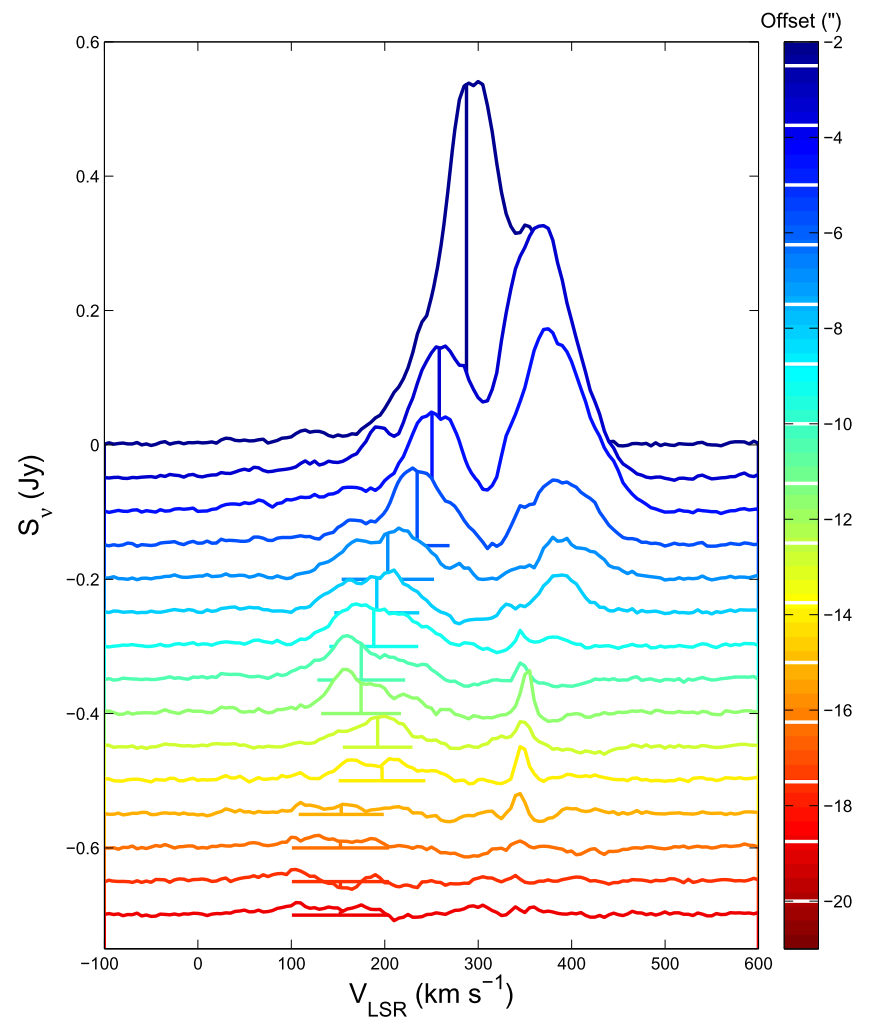

Figure 3. $\mathrm{CO}(1-0)$ spectra along the southern part of the position-velocity cut in Figures 1 and 2, following the SW streamer. The colors indicate offsets from the plane, as indicated by the color bar to the right (spectra are offset vertically by 0.05 Jy for display purposes). At small offsets, the emission is dominated by the starburst in the central bar, and line splitting is observed for intermediate offsets $\left(-4^{\prime \prime}\right.$ to $\left.-10^{\prime \prime}\right)$. The diffuse molecular outflow is then picked up at $v_{\mathrm{lsr}} \sim$ $200 \mathrm{~km} \mathrm{~s}^{-1}$, and can be traced out to offsets of $-18^{\prime \prime}$ (the horizontal and vertical bars indicate the Gaussian-fit FWHM, central velocity, and amplitudes in the streamer). Given the low $\mathrm{S} / \mathrm{N}$ of the three spectra at the largest offsets, we do not use their fits in our subsequent analysis. The narrow $\mathrm{CO}(1-0)$ feature around $v_{\mathrm{lsr}} \sim 350 \mathrm{~km} \mathrm{~s}^{-1}$ is due to molecular gas emission from a GMC in the disk of NGC 253, labeled in Figure 2 as "disk cloud."

The Gaussian fitting results are summarized in Figure 4. The left panel shows the central velocity and FWHM velocity width $(\Delta v)$ of the emission as a function of position along the $p v$ diagram. A first-order polynomial fit to the central velocity as a function of position is overplotted as a red line. The right panel shows the velocity and deconvolved (by subtracting the beam size in quadrature) FWHM spatial width as a function of position: the outflow has a deconvolved FWHM width of approximately $40-50 \mathrm{pc}$. The reference velocity is indicated by a dashed white line in Figure 2. It corresponds to the approximate gas velocity of the bar at the base of the outflow.

\subsection{Is the Molecular Outflow Accelerating?}

The $S W$ streamer is blueshifted with respect to the reference velocity, implying that the material is approaching us. This blueshift gets larger with increasing distance from the galaxy plane, which can be interpreted as an accelerating continuous outflow. This interpretation of the data is, however, not unique. If the original ejection occurred over a short period of time and had a distribution of velocities, the fast ejecta would have gotten farther away than the slower ejecta, giving rise to a velocity gradient along the streamer. The apparent projected velocity gradient could also be due to a gradient in the inclination of the outflow cone, with the (more or less) constant velocity of $\mathrm{CO}$ gas becoming increasingly aligned with the line of sight at farther distances from the central bar. Such geometry has been reported in the ionized gas of the bubble of NGC 3079 (e.g., Veilleux et al. 1994; Cecil et al. 2001). The ionized outflow of NGC 253, however, seems more like an open-ended cone than a bubble (Westmoquette et al. 2011), suggesting that this explanation is probably less likely.

The translation of observed line-of-sight velocities into actual velocities is crucial to determine the mass outflow rate and whether the material escapes the galaxy or not. The inclination of the galaxy is $78^{\circ}$ (Westmoquette et al. 2011), so the projected edge of the southern outflow cone will have an inclination of $78^{\circ}-90^{\circ}=-12^{\circ}$, where the negative sign indicates that it is pointing toward us, resulting in blueshifted emission. If the emission originates precisely from this projected edge, the $\sin i$ correction would be very large (a factor of $\sim 5$ ). Based on the median of the distribution of $\tan i$ for the surface of a cone with the opening angle and orientation as determined by Westmoquette et al. (2011) for the ionized gas outflow, Bolatto et al. (2013) argue that a correction factor of approximately three is more likely, and we adopt that value here. Note that, in practice, the actual factor can range from $\sim 1$ to $\infty$ so the projection correction has large uncertainties.

We measure a gradient of $d v / d r=6.1 \mathrm{~km} \mathrm{~s}^{-1} /\left[^{\prime \prime}\right]$ or $\sim 36 \mathrm{~km} \mathrm{~s}^{-1} /[100 \mathrm{pc}]$ from the polynomial fitting shown in Figure 4 (the symmetric ordinary-least-squares bisector yields a $25 \%$ higher value). Adopting the aforementioned projection correction factor of three, which includes both the corrections for distances and velocities, we derive an actual velocity gradient of $\sim 100 \mathrm{~km} \mathrm{~s}^{-1} /[100 \mathrm{pc}]$, or $\sim 1 \mathrm{~km} \mathrm{~s}^{-1} /[\mathrm{pc}]$. Toward the end of the detected outflow, we measure a line-of-sight outflow speed, relative to our reference disk velocity, of $120 \mathrm{~km} \mathrm{~s}^{-1}$. With the projection correction, this translates into an actual outflow speed of $\sim 360 \mathrm{~km} \mathrm{~s}^{-1}$. The escape velocity of NGC 253 can be approximated using Equation (16) in Rupke et al. (2002), and the circular velocity by HlavacekLarrondo et al. (2011). This yields an escape velocity of $\sim 500 \mathrm{~km} \mathrm{~s}^{-1}$ (with significant error bars), i.e., the measured outflow velocity is approaching the escape velocity.

The velocity dispersion and width of the SW streamer are consistent with the dynamical age of the feature. The 

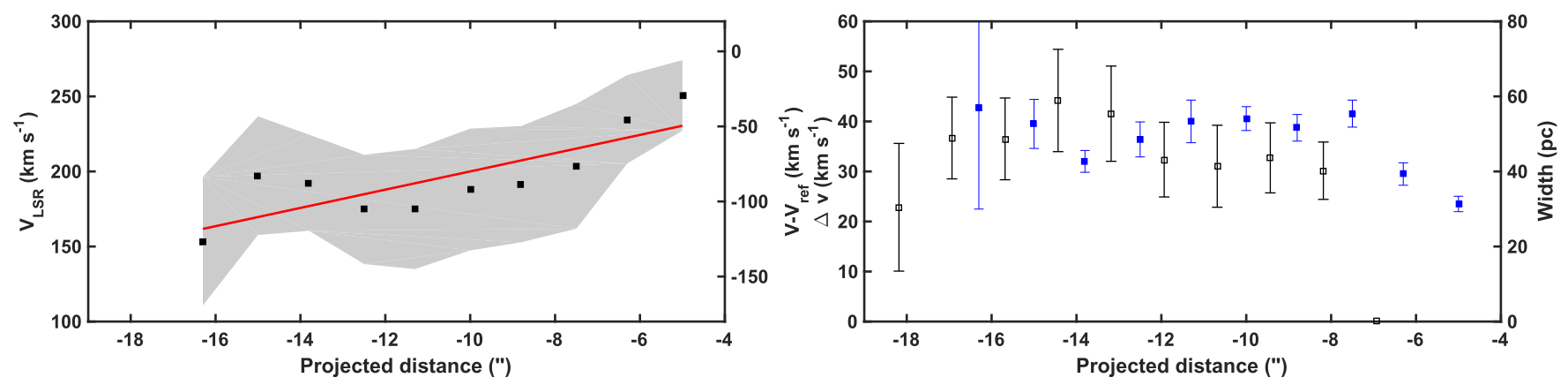

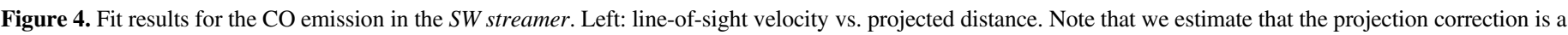

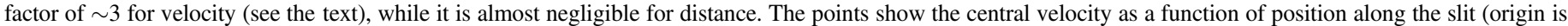

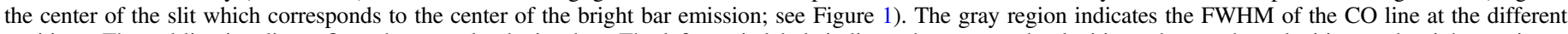

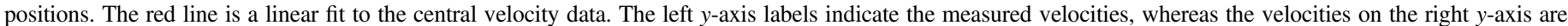

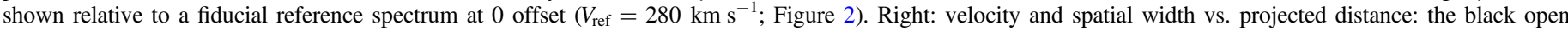

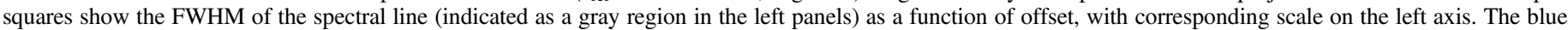

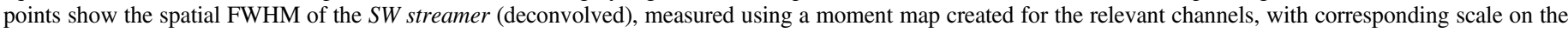
right axis.
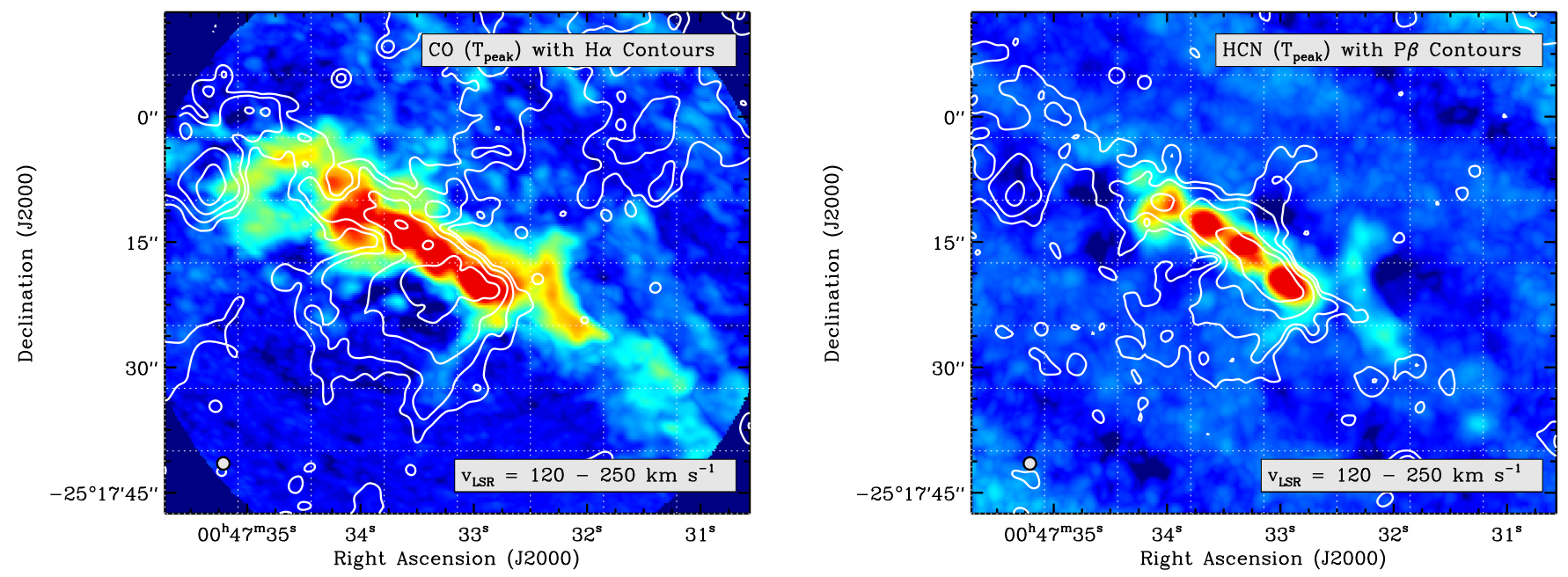

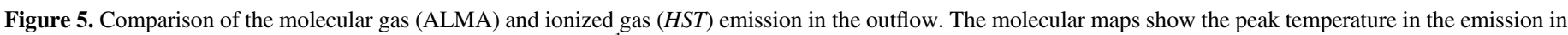

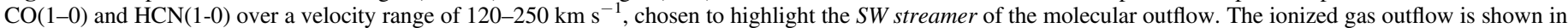

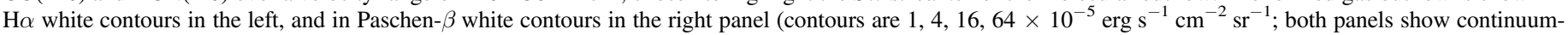
subtracted HST observations). The coordinate grid is overlaid to facilitate comparison. The SW streamer is already clearly apparent in HCN emission.

dynamical age of the streamer is $\sim 1 \mathrm{Myr}$, corresponding to the approximate ratio of its deprojected speed of $\sim 300 \mathrm{~km} \mathrm{~s}^{-1}$ and length of $\sim 300 \mathrm{pc}$. The measured velocity dispersion $\Delta v \sim 35-40 \mathrm{~km} \mathrm{~s}^{-1}$, which would naturally produce a structure with a width of $\sim 40 \mathrm{pc}$ in $1 \mathrm{Myr}$, very similar to the measured physical width (Figure 4). If the feature were significantly older than $1 \mathrm{Myr}$, it would have to be confined (by external pressure or magnetic fields) to stay as narrow in spatial extent as observed. This implies that, unless such confinement is present, the outflow velocities cannot be much slower than $300 \mathrm{~km} \mathrm{~s}^{-1}$.

\subsection{Deep Imaging of the Ionized Gas}

Figure 5 compares the outflow observed in molecular gas (left: $\mathrm{CO}$, right: $\mathrm{HCN}$ ) to the well-known ionized gas outflow (left: $\mathrm{H} \alpha$, right: $\mathrm{Pa} \beta$ ). The tracers of the ionized outflow shown here $(\mathrm{H} \alpha$ and $\mathrm{Pa} \beta)$ are significantly affected by extinction toward the northern part of the galaxy. The ALMA images correspond to peak intensity over the range of $v=120-250 \mathrm{~km} \mathrm{~s}^{-1}$, highlighting the disk, eastern shell, and western outflow. The $S W$ streamer aligns closely with the edge of the outflow as seen in both $\mathrm{H} \alpha$ and the more extinctionrobust $\mathrm{Pa} \beta$ imaging (contours). In fact, the streamer aligns with bright features in both recombination lines, further solidifying the association of this feature with the ionized gas outflow. The higher density of gas near the cold streamer presumably leads to a higher emission measure and corresponding emissivity of ionized gas. We note that the feature seen in dense gas emission on the other side of the disk (i.e., opposite to the $S W$ streamer) is likely due to peculiar motions in the NGC 253's disk. This region was already identified in early high-density tracer observations using OVRO (Knudsen et al. 2007).

The molecular component seems to confine the ionized features in the left panel of Figure $5(\mathrm{CO}+\mathrm{H} \alpha)$, with the ionized gas tapering to emerge from the center of the circumnuclear starburst. As noted previously, both recombination lines are brighter to the south of the galaxy, consistent with that being the approaching side of the outflow. Strikingly, extinction due to the surrounding disk is strong enough that aside from one bright pillar, even $\mathrm{Pa} \beta$ emission is not visible north of the starburst region.

Perhaps surprisingly, there is little recombination line emission (and thus little sign of very recent massive star 


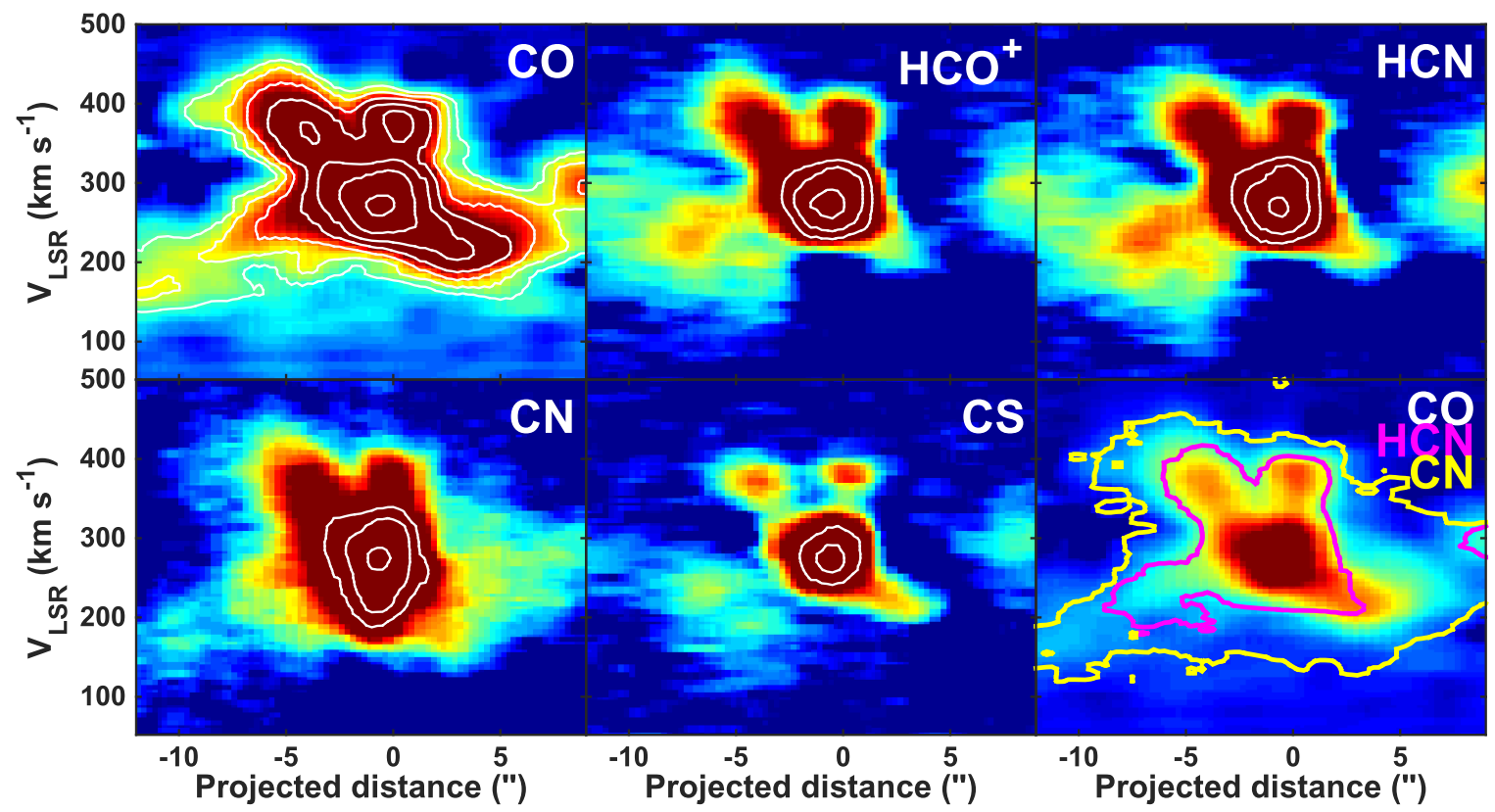

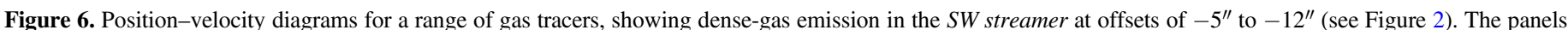

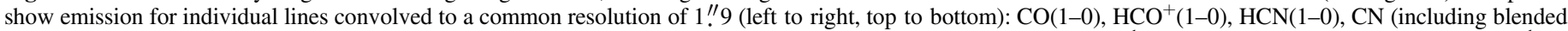

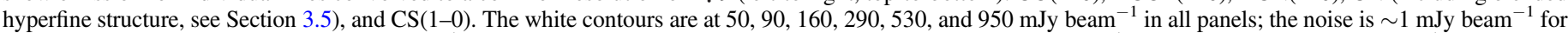

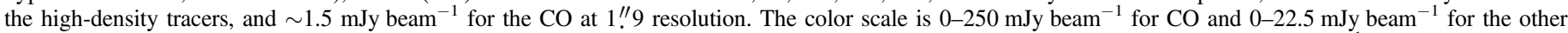

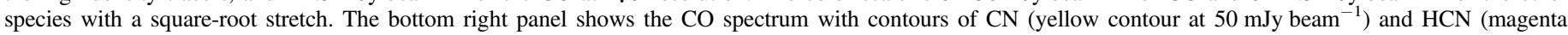

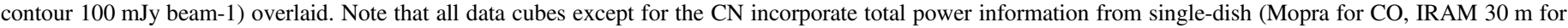
the rest, see Section 2.1.2).

formation) in the far western part of the disk. Also, we do not see strong emission centered on the putative expanding molecular shells identified in Sakamoto et al. (2006). Bolatto et al. (2013) argued that these shells are associated with the molecular streamers in the outflow and are presumably driven by young stellar clusters of $M_{*} \sim 10^{5} M_{\odot}$. If the shells are driven by stellar feedback, the population driving them must be either older than $\sim 5 \mathrm{Myr}$ or so embedded as to remain inconspicuous in the $\mathrm{Pa} \beta$ image.

\subsection{Dense Gas in the Outflow}

From Figure 5, we conclude that $\mathrm{HCN}(1-0)$ and $\mathrm{CO}(1-0)$ emission is present along the $S W$ streamer in the relevant velocity range of $120-250 \mathrm{~km} \mathrm{~s}^{-1}$. In Figure 6, we show the position-velocity diagrams for high-dipole molecules (HCN, $\mathrm{HCO}^{+}, \mathrm{CS}$, and $\mathrm{CN}$ ) associated with dense gas and compare them to the $\mathrm{CO}$ emission ( $\mathrm{CN}$ is usually the chemical byproduct of the photodissociation of $\mathrm{HCN}$ ). It is clear from Figures 5 and 6 that the dense gas tracers also show the same outflow characteristics apparent in $\mathrm{CO}(1-0)$, at position offsets $<-5^{\prime \prime}$ and line-of-sight velocities of $<300 \mathrm{~km} \mathrm{~s}^{-1}$. HCN and $\mathrm{HCO}^{+}$emission in an outflow have, to date, only been reported in a few systems: these include the ULIRG QSO Mrk 231 (Aalto et al. 2012; Lindberg et al. 2016), a system with $\mathrm{SFR} \sim 200 M_{\odot} \mathrm{yr}^{-1}, 70$ times larger than that of NGC 253. In NGC 1266, velocity wings are detected in $\mathrm{CS}(2-1)$ and $\mathrm{HCN}$ (1-0) (Alatalo et al. 2015). In NGC 1068, García-Burillo et al. (2014) report dense molecular gas detections in an AGN-driven outflow. In the starburst galaxy M82, the detection of dense gas tracers at the base of its molecular outflow (Walter et al. 2002; Leroy et al. 2015a) have been reported by Salas et al. (2014).

The clear detection of dense gas tracers in the $S W$ streamer of NGC 253 provides an interesting new constraint on small spatial scales of outflowing gas. Note that the $\mathrm{CN}$ emission suffers from artificial velocity broadening due to blending of hyperfine components: the brightest $\mathrm{CN}$ hyperfine component $(1,3 / 2,5 / 2 \rightarrow 0,1 / 2,3 / 2)$ is at $113490.9 \mathrm{MHz}$, and the two brightest satellites (a factor of three fainter) are $(1,3 / 2,1 / 2 \rightarrow$ $0,1 / 2,1 / 2)$ at $113499.6 \mathrm{MHz}$ and $(1,3 / 2,3 / 2 \rightarrow 0,1 / 2,3 / 2)$ at $113508.9 \mathrm{MHz}$, corresponding to $24 \mathrm{~km} \mathrm{~s}^{-1}$ and $50 \mathrm{~km} \mathrm{~s}^{-1}$ blueward of the main component (see Skatrud et al. 1983 for $\mathrm{CN}$ transitions and rest frequencies).

We quantify the relation between the different transitions observed in the streamer using Figure 7 , in which we present spectra in $\mathrm{CO}, \mathrm{HCN}, \mathrm{HCO}^{+}, \mathrm{CS}$, and $\mathrm{CN}$ at different offsets from the center (as before, all data are on a common resolution of 1."9). These spectra show both emission from the molecular gas streamer (at velocities $\sim 200 \mathrm{~km} \mathrm{~s}^{-1}$ ) as well as emission from the disk of NGC $253\left(\sim 400 \mathrm{~km} \mathrm{~s}^{-1}\right)$, which allows us to immediately compare their line ratios. In the central region, we measure peak flux densities for $\mathrm{CO}, \mathrm{CN}, \mathrm{CS}, \mathrm{HCN}$, and $\mathrm{HCO}^{+}$ of $1548.0,139.8,66.3,112.6$, and $123.5 \mathrm{mJy} \mathrm{beam}^{-1}$. Consequently, we measure a flux ratio of $\sim 13.8$ between $\mathrm{CO}$ and $\mathrm{HCN}$, which corresponds to a Rayleigh-Jeans brightness temperature ratio of $\sim 8$. Such ratios, indicating a high dense gas fraction, is typical of starburst environments (e.g., Gao \& Solomon 2004) and also seen in some of the densest regions of nearby starforming disks (e.g., Usero et al. 2015).

At an offset of -6.5 , significantly off the central starburst region and clearly corresponding to the kinematically distinct $S W$ streamer, we measure the following values for the outflow feature at $\sim 200 \mathrm{~km} \mathrm{~s}^{-1}$ (in the same order and units as above): 154.0, 9.8, 7.0, 12.8, and 10.4. These values yield a $\mathrm{CO} / \mathrm{HCN}$ peak flux density ratio of $\sim 12$. This is very similar to the value of the $\mathrm{CO} / \mathrm{HCN}$ ratio in the starburst, and the same holds true for ratios of $\mathrm{CO}$ to the other molecules. By contrast, the corresponding values for the emission that we kinematically 


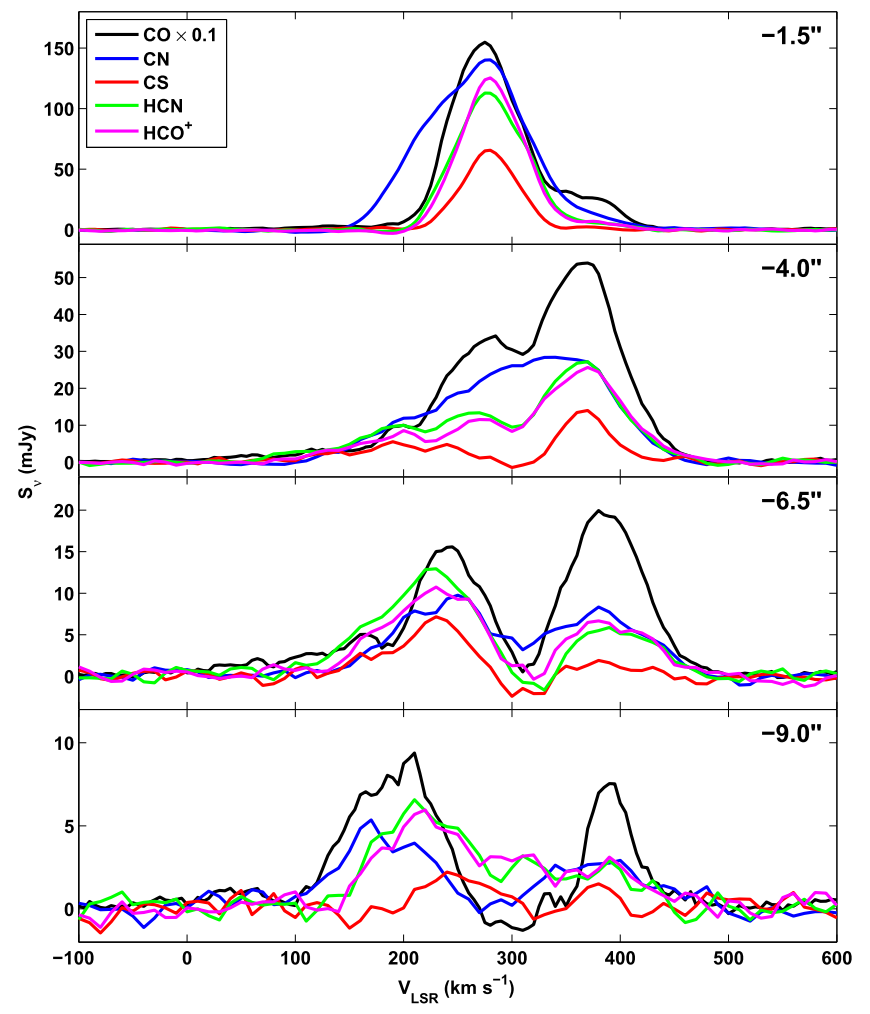

Figure 7. Spectra along the southern outflow at different offsets from the center (shown in the top right of each panel in arcsec, " 0 " offset corresponds to the plane of the disk). The black lines indicate the $\mathrm{CO}$ emission (scaled down by a factor of 10 for display purposes), and the $\mathrm{CN}, \mathrm{CS}, \mathrm{HCN}$, and $\mathrm{HCO}+$ spectra are shown in blue, red, green, and magenta, respectively (all data have been convolved to the same beam of $1{ }^{\prime \prime} 9$ ). Note that the CN line is artificially broadened by the blended hyperfine structure (see Section 3.5). The feature at $\sim 200 \mathrm{~km} \mathrm{~s}^{-1}$ is associated with the $S W$ streamer, and the emission at $\sim 400 \mathrm{~km} \mathrm{~s}^{-1}$ is from the disk. Note how the ratio of $\mathrm{CO} /$ (dense gas tracer) is much lower in the outflow (and similar there to the central starburst region at offsets -1 !.5) than in the disk, suggesting that dense clumps with properties similar to the dense gas in the starburst (and of much larger density than gas in typical disk GMCs) survive in the outflow.

identify with the background rotating disk component of the galaxy (visible at a velocity of $\sim 400 \mathrm{~km} \mathrm{~s}^{-1}$ in Figure 7) are 193, $8.4,1.9,5.6$, and 6.4 (same order and units as above). These yield a $\mathrm{CO} / \mathrm{HCN}$ peak flux density ratio $\sim 35$, a factor of three higher than the starburst or the streamer. Such a value for the $\mathrm{CO} / \mathrm{HCN}$ ratio is in excellent agreement with typical measurements in the disks of nearby galaxies (Usero et al. 2015). We conclude that the fraction of dense molecular gas in the SW streamer of the outflow is high and stays high throughout the region where we detect emission. Moreover, the material in the molecular streamer displays a dense gas fraction that is similar to that of the starburst region and significantly higher than that of the main disk of NGC 253, strongly suggesting a starburst origin for the gas in the streamer.

\subsection{Mass and Density of the SW Streamer}

A key result in the study of Meier et al. (2015) is that the $\mathrm{CO}(1-0), \mathrm{HCN}(1-0)$, and $\mathrm{HCO}^{+}(1-0)$ transitions are optically thick (and, in fact, have similar optical depths, $\tau \sim 5$ ) in the central region of NGC 253. The fact that the line ratios we measure do not change from those in the central region suggests that their emission is also optically thick in the $S W$ streamer. This has important potential consequences for the molecular gas mass in the outflow. The $\mathrm{CO}(1-0)$ luminosity of the streamer is $L_{\mathrm{CO}} \sim 2.8 \times 10^{6} \mathrm{~K} \mathrm{~km} \mathrm{~s}^{-1} \mathrm{pc}^{2}$, with an approximate area of $\sim 1.1 \times 10^{4} \mathrm{pc}^{2}$ and an integrated surface brightness of $T_{\mathrm{CO}} \Delta v \sim 250 \mathrm{~K} \mathrm{~km} \mathrm{~s}^{-1}$. In order to obtain a firm lower limit for its mass, Bolatto et al. (2013) used a CO-to- $\mathrm{H}_{2}$ conversion factor of $\alpha_{\mathrm{CO}}=0.34 M_{\odot}\left(\mathrm{K} \mathrm{km} \mathrm{s}^{-1} \mathrm{pc}^{2}\right)^{-1}$ based on optically thin $\mathrm{CO}(1-0)$ emission. This leads to a "minimum mass" of the streamer of $\sim 10^{6} M_{\odot}(\sim 13$ times lower than what one would derive with the typically assumed Galactic conversion factor).

\subsubsection{Inferred Volume Densities and Implications for Dense Gas Excitation}

The projected area of the streamer is $\sim 240 \times 60 \mathrm{pc}^{2}$ (or $\sim 10^{41} \mathrm{~cm}^{2}$ ), leading to an average $\mathrm{H}_{2}$ column density of $N\left(\mathrm{H}_{2}\right) \sim 4 \times 10^{21} \mathrm{~cm}^{-2}$ in the plane of the sky. If we assume that the feature has cylindrical geometry (depth $\sim$ width) this results in an average volume density of $40 \mathrm{~cm}^{-3}$. This is completely insufficient to excite the detected dense gas tracers through collisions: for example, the critical density (defined as the density at which the rate of the collisional depopulation of a quantum level equals the spontaneous radiative decay rate) for the $\operatorname{HCN}(1-0)$ emission is $2.6 \times 10^{6} \mathrm{~cm}^{-3}$. As is frequently pointed out, however, what is more relevant to the excitation is the "effective excitation density," which includes the summation of all collisional transitions to the lower level and the effect of radiative trapping in an optically thick environment (e.g., Stacey 2011; Shirley 2015). The effective excitation density can be one to two orders of magnitude lower than the critical density even for HCN, particularly in environments of high optical depth (Shirley 2015). Nonetheless, even after taking these effects into account, our derived average gas density would be still two to three orders of magnitude too low to explain the presence of bright emission from $\mathrm{HCN}$ and other high-dipole molecules. Note also that the line-widths are large (Figure 7), which lowers the effect of radiative trapping.

There are a number of factors that could bring these numbers in agreement. First of all is the clumping - the molecular gas is likely not distributed smoothly. The observed surface brightness in the $S W$ streamer at $32 \mathrm{pc}$ resolution is $T_{\mathrm{b}}^{\mathrm{obs}} \sim 1.5-2 \mathrm{~K}$. Assuming an intrinsic brightness of $T_{\mathrm{b}} \sim 100 \mathrm{~K}$ (not unreasonable since the gas in the streamer is likely warm), the resulting area clumping factor would be $\sim T_{\mathrm{b}} / T_{\mathrm{b}}^{\mathrm{obs}} \approx 50-70$, corresponding to a clumping factor in volume of 503/2 $~ 350-600$. This implies that our bulk density of $40 \mathrm{~cm}^{-3}$ is physically closer to $2 \times 10^{4} \mathrm{~cm}^{-3}$, with typical column densities of $N$ $\left(\mathrm{H}_{2}\right) \sim 10^{22} \mathrm{~cm}^{-2}$. Note that these estimates come from the "minimum mass" yielded by the assumption of an optically thin CO-to- $\mathrm{H}_{2}$ conversion factor. Depending on the optical depth of the $\mathrm{CO}(1-0)$ transition, the actual conversion factor (and, consequently, the derived molecular mass and resulting mass outflow rate) could be factors of a few to several times higher. To produce bright $\mathrm{HCN}$ emission it would be more comfortable to raise the mass in the streamer by a factor of a few, which would raise the physical volume density to $10^{5} \mathrm{~cm}^{-3}$ and reach conditions where $\mathrm{HCN}(1-0)$ would be efficiently excited. Following this line of reasoning, the bright $\mathrm{HCN}$ emission in the streamer suggests that the minimum molecular outflow rate of $3 M_{\odot} \mathrm{yr}^{-1}$ is probably an underestimate by factors of a few (Bolatto et al. 2013). 


\subsubsection{Excitation by Electrons?}

Collisions with electrons are also an effective way of exciting polar molecules (Dickinson et al. 1977). In fact, they are the main mechanism for HCN excitation in comets (Lovell et al. 2004). Calculations show that the rate coefficient for excitation of $\mathrm{HCN}(1-0)$ by collisions with electrons is $>10^{5}$ times larger than for collisions with $\mathrm{H}_{2}$ (Saha et al. 1981; Faure et al. 2007). The reason why electrons are usually not considered as important collisional partners for $\mathrm{HCN}$ is that the electron fraction, $x_{e}$, in dark molecular clouds is thought to be $x_{e} \sim 10^{-8}$ or lower, controlled by cosmic-ray ionization (e.g., Flower et al. 2007). However, for $x_{e} \gtrsim 10^{-6}$ excitation by collisions with electrons will be important and likely dominant. The $S W$ streamer is close to and probably embedded in the ionized flow, so it is possible that it possesses a higher ionization fraction than a typical dark cloud, and $\mathrm{HCN}(1-0)$ is mostly excited by electrons. This could also be the case if, for example, the outflow is cosmic-ray driven. We note, however, that the line ratios in the $S W$ streamer are very similar to the line ratios observed in the starburst (Figure 7), which makes it unlikely that the excitation mechanisms that dominate in one region do not also dominate in the other.

\subsubsection{Constraints to Mass and Surface Density from Extinction}

We can use the extinction inferred for the $S W$ streamer from the observed $\mathrm{H} \alpha$ to $\mathrm{Pa} \beta$ line ratio to independently constrain its molecular mass. The typical $\mathrm{H} \alpha / \mathrm{Pa} \beta$ ratio in the streamer is $R_{\text {obs }}=4.5-5$, while the expected intrinsic line ratio in a $10^{4} \mathrm{~K}$ plasma is $R_{\text {int }} \approx 17.5$ for case B recombination (Osterbrock \& Ferland 2006). For a Cardelli et al. (1989) extinction curve, the ratio of extinction at $1.28 \mu \mathrm{m}$ to extinction at $0.66 \mu \mathrm{m}$ is $r_{P \beta \mathrm{H} \alpha}=A_{\mathrm{Pa} \beta} / A_{\mathrm{H} \alpha} \simeq 1 / 3$. Thus explaining the observed line ratio with a single screen of extinction in front of the ionized gas requires $A_{\mathrm{H} \alpha}=-2.5 \log \left[R_{\mathrm{obs}} / R_{\text {int }}\right] /\left(1-r_{P \beta \mathrm{H} \alpha}\right) \approx$ $2-2.2$, which results in $A_{V} \sim 2.5$. For the Milky Way, this implies a molecular column density in the plane of the sky of $N\left(\mathrm{H}_{2}\right) \sim 2.5 \times 10^{21} \mathrm{~cm}^{-2}$ (Bohlin et al. 1978). The screen geometry is a lower limit to the column density, since any unextincted ionized gas emission in front of the screen leads to an increase in $R_{\mathrm{obs}}$, while conversely any extinction on the back side of the ionized emission would remain undetected. To account for some of these effects a "double screen" geometry is usually preferred, leading to $N\left(\mathrm{H}_{2}\right) \sim 5 \times 10^{21} \mathrm{~cm}^{-2}$. We would reach a $20 \%$ higher value if we assumed that the dust was mixed with the gas. These numbers are consistent with the "lower limit" mass estimate from CO inferred above. Optically thin $\mathrm{CO}$ emission, however, is somewhat surprising given the relatively low $\mathrm{CO} / \mathrm{HCN}$ ratio in the streamer, reminiscent of the central starburst region. Thus it is likely that there is significant ionized gas in front of the streamer. ALMA observations of higher- $J$ CO lines will help to constrain the properties of the $S W$ streamer and the conditions in the molecular outflow further.

\subsection{The Driving of the SW Streamer}

The mechanisms to impart momentum and accelerate cold gas in a galactic outflow are not well understood. The presence of molecular material $\gtrsim 300 \mathrm{pc}$ away from the central bar, particularly in the form of dense gas as traced by $\mathrm{HCN}, \mathrm{HCO}^{+}$, and CS, already provides strong constraints on the stability of wind-entrained clouds against photo and thermal evaporation,
Kelvin-Helmoltz instabilities, and shedding due to ablation (e.g., Marcolini et al. 2005). Simulations of radiative clouds embedded in a supersonic flow show that radiative cooling stabilizes clouds against destruction (Cooper et al. 2009). Radiative clouds undergo a lower acceleration and have a higher Mach number relative to the flow than adiabatic (energyconserving) clouds. They do experience fragmentation due to Kelvin-Helmholtz instability, but the resulting cloudlets are denser and are more resistent to destruction than adiabatic clouds, and they are drawn into the flow creating filaments. Additionally, magnetized clouds entrained in a hot wind have also been shown to be more stable than unmagnetized ones, even for very moderate initial internal magnetic fields, while also leading to filamentary structures (McCourt et al. 2015). In a set of recent calculations, Scannapieco \& Brüggen (2015) show that supersonic flows suppress the Kelvin-Helmholtz instability, thus allowing cooling clouds to survive longer, but the compression of the cloud also makes its acceleration less efficient so that they only reach $\sim 15 \%$ of the hot flow velocity before being disrupted. The inclusion of cloud evaporation induces further compression and naturally produces highly elongated, filamentary clouds similar to the streamers we observe, but, though it can help the clouds survive even longer, it also makes the momentum transfer less efficient, resulting in even lower final velocities before disruption (Brüggen \& Scannapieco 2016). Very recent simulations by McCourt et al. (2016), however, suggest that crushed clouds shatter into tiny, dense, cloudlets that do no disappear and can be much more easily accelerated by the hot wind, making the entrainment process efficient. In this scenario, the $S W$ streamer could be composed of a collection of dense molecular clumps embedded in the much hotter outflow.

Notably, a feature corresponding to the molecular gas streamer is also seen in radio continuum maps obtained at $20 \mathrm{~cm}$ (Figure 9 in Ulvestad \& Antonucci 1997, see also Heesen et al. 2009), implying that synchrotron emission is associated with the outflowing molecular gas. This suggests that magnetic fields, and possibly cosmic rays, are associated with the outflow. Cosmic rays have been suggested as an important mechanism for driving outflows in galaxies (e.g., Booth et al. 2013; Salem \& Bryan 2014; Girichidis et al. 2016; Simpson et al. 2016), although observationally constraining their importance remains elusive.

To impart momentum to the cold gas, an alternative to entrainment in a hot flow is acceleration by radiation pressure. In order to explore the viability of radiation pressure to explain the observations, we perform a rough calculation. The radiation flux at a distance $r$ along its axis from the center of a uniformbrightness disk of radius $R_{\mathrm{src}}$ and luminosity $L_{\mathrm{src}}$ is $F=L_{\text {src }} /\left[2 \pi\left(R_{\text {src }}^{2}+r^{2}\right)\right]$. Only the photons absorbed by the dusty cold gas can impart momentum, so the force due to radiation pressure experienced by a cloud that subtends a solid angle $\Omega$ and has an optical depth $\tau$ is $f_{\text {rad }}=$ $F \Omega r^{2}\left(1-e^{-\tau}\right) / c$. The resulting velocity is then simply $v_{\text {cloud }}^{2}=2 \int f_{\text {rad }} / M_{\text {cloud }} d r$. For the purposes of the calculations below, we will assume that the $S W$ streamer is exposed to radiation from the "naked" starburst, which would be mostly far-ultraviolet and easily absorbed $(\tau \gg 1)$. There is the potential for the momentum imparted by the radiation to be boosted by a factor of the order of $\tau_{\mathrm{IR}}$, the mean infrared optical depth of the streamer (e.g., Thompson et al. 2015; Zhang \& Davis 2016). Given our column density estimates, the $S W$ 
streamer does not have a large enough column to possess substantial optical depth to its own reradiated IR photons ( $\tau_{\text {IR }} \sim 0.2$ for the most favorable geometry), so we assume that this boosting is unimportant.

NGC 253 has a total infrared luminosity of $\mathrm{L}_{\mathrm{TIR}}=$ $1.4 \times 10^{44} \mathrm{erg} \mathrm{s}^{-1}$, about half of which originates within a diameter of $\sim 200$ pc from its center (Leroy et al. 2015b), i.e., $R_{\text {src }}=100$ pc. The $S W$ streamer has a "minimum mass" $M_{\text {cloud }} \approx 10^{6} M_{\odot}$, a projected size on the sky of $\sim 60 \times 240$ $\mathrm{pc}^{2}$, and a typical distance to the center of the order of $r \sim 100$ pc. If we assume that the geometry corresponds to a cylinder $60 \mathrm{pc}$ in diameter $\left(\Omega r^{2}=\pi(30 \mathrm{pc})^{2}\right)$ illuminated by $L_{\text {src }}=10^{44} \mathrm{erg} \mathrm{s}^{-1}$, the force due to radiation pressure applied at the base of the streamer would be $f_{\text {rad }} \sim 7.5 \times 10^{31}$ dyn, resulting in an estimated acceleration of $a_{\mathrm{rad}} \sim$ $4 \times 10^{-8} \mathrm{~cm} \mathrm{~s}^{-2}$. In the absence of gravity, the resulting velocity at $r \sim 100 \mathrm{pc}$ would be $v_{\text {cloud }} \sim 60 \mathrm{~km} \mathrm{~s}^{-1}$, lower than the observed velocity before projection correction (Figure 4). If we instead assume that the radiation is exerting pressure on the long side of the cylinder (the geometry with the maximum area), $f_{\text {rad }}$ would be five times larger, with a corresponding $v_{\text {cloud }} \sim 135 \mathrm{~km} \mathrm{~s}^{-1}$. Note, however, that the ratio of gravity to radiation pressure for stars is $f_{\text {grav }} / f_{\text {rad }}=G \Sigma_{\text {cloud }}(4 \pi c) / \Psi$, where $\psi \approx 2000 \mathrm{erg} \mathrm{s}^{-1} \mathrm{~g}$ for a fully sampled IMF containing young stars, and $\Sigma_{\text {cloud }}$ is the surface density of the cloud ( $\Psi$ is the light-to-mass ratio of the stellar population, e.g., Equation (33) in Kim et al. 2016). For the end-on geometry $\Sigma_{\text {cloud }} \sim 0.075 \mathrm{~g} \mathrm{~cm}^{-2}$, while for the sideon geometry $\Sigma_{\text {cloud }} \sim 0.015 \mathrm{~g} \mathrm{~cm}^{-2}$, resulting in $0.94 \geqslant f_{\text {grav }} / f_{\text {rad }} \geqslant 0.19$. So, for our assumed geometry and mass, the net acceleration $a_{\text {net }}=a_{\text {rad }}\left(1-a_{\text {grav }} / a_{\text {rad }}\right)$ is between $6 \%$ and $81 \%$ of $a_{\mathrm{rad}}$, which would result in $15 \leqslant v_{\text {cloud }} \leqslant 120 \mathrm{~km} \mathrm{~s}^{-1}$.

From the above estimates, it appears that radiation can contribute maybe up to a few tens of percent of the momentum, but it is extremely unlikely to completely explain the observations. Doing so would require using the most favorable geometric assumption (maximal cloud area, which maximizes $f_{\text {rad }}$ and minimizes $\left.f_{\text {grav }} / f_{\text {rad }}\right)$ and also assuming that there is almost no projection correction to the measured velocity (which requires a contrived geometry). This suggests that radiation pressure is not the dominant mechanism for accelerating the streamer, though it is non-negligible. Note also that any increase in the mass of the streamer over its minimum mass (see discussion in Section 3.6.1), or accounting for the old stars in the galaxy disk outside the starburst (which would increase gravity), would also reduce the importance of radiation pressure. Much higher resolution observations of the SW streamer may help further elucidate these questions, in particular, which mechanisms are driving the molecular gas in the outflow.

\section{Summary}

Our new ALMA band 3 observations ( $\mathrm{CO}$ and dense gas tracers) of the central starburst region of NGC 253 give new insights on the properties of the molecular outflowing gas originally discussed in Bolatto et al. (2013). The most prominent outflow feature toward the south, which is the main subject of this paper, possesses a large intrinsic velocity dispersion. Its extent and dispersion are consistent with an ejection from the disk starting about $\sim 1$ Myr ago.
It is currently unclear whether or not the molecular mass entrained in the outflow will escape the galaxy or be recycled to fuel later episodes of star formation. For most of the gas in the observed outflow to escape the galaxy, it would need to be accelerated as it moves away from the disk. The kinematics of the molecular gas are consistent with accelerating with a velocity gradient of $1 \mathrm{~km} \mathrm{~s}^{-1} \mathrm{pc}^{-1}$, and at its last measurable point it approaches the escape velocity. As discussed, this interpretation is not unique: the kinematics could also be consistent with an outflow with a range of speeds, where the material farther from the disk is there because it is the fastest. In that scenario, only the fastest fraction of the outflowing gas may escape the galaxy. Approved, more sensitive ALMA observations will trace the outflow even further out and will shed light on whether or not part of the ejected molecular material will escape the galaxy.

Strikingly, tracers of the dense gas phase of the molecular medium ( $\mathrm{HCN}, \mathrm{HCO}^{+}, \mathrm{CS}$, and $\mathrm{CN}$ ) are also spatially coincident with the $S W$ streamer of the molecular outflow. The line ratios $\mathrm{HCN} / \mathrm{CO}$ of $\sim 1 / 10$ measured in the outflow are high and consistent with ratios observed in the central starburst region of NGC 253 and in other starbursts. The HCN/ $\mathrm{CO}$ line ratio in the disk, on the other hand, is significantly lower $(\sim 1 / 30)$, typical of gas in the disks of nearby galaxies. In principle, this is indicative of the dense molecular gas being ejected from the central regions into the outflow, while retaining its properties in this process. It also suggests that the $\mathrm{CO}(1-0)$ and $\mathrm{HCN}(1-0)$ emission are also optically thick in the streamer, implying that the estimated mass loading parameter $\eta \gtrsim 3$ (Bolatto et al. 2013) is likely a lower limit. Note that electron excitation of polar molecules is an oftenignored mechanism that could play an important role at exciting $\mathrm{HCN}, \mathrm{HCO}^{+}$, and $\mathrm{CS}$ emission in outflows, though the fact that we see the same ratios of these transitions to $\mathrm{CO}(1-0)$ in the starburst and the streamer suggests similar excitation mechanisms in both regions, implying dense gas is the most likely cause. Simple calculations indicate that radiation pressure is not the main mechanism for driving the outflow. The presence of a dense gas phase in molecular outflows (with volume densities $>10^{4} \mathrm{~cm}^{-3}$ and probably $\sim 10^{5} \mathrm{~cm}^{-3}$ ) will have to be accounted for in numerical simulations of galactic winds, both at low and high redshift.

We thank the referee for excellent comments that improved the paper. D.S.M. acknowledges partial support by the National Science Foundation through grant AST-1009620. S.V. acknowledges NSF grant AST-1009583. A.D.B. acknowledges visiting support by the Alexander von Humboldt Foundation, and support by the National Science Foundation through a CAREER grant AST-0955836 and AST-1412419. E.C.O. is supported by the National Science Foundation through grant AST-1312006. This paper makes use of the following ALMA data: ADS/JAO.ALMA \#2011.0.00172.S, \#2012.1.00108.S. ALMA is a partnership of ESO (representing its member states), NSF (USA), and NINS (Japan), together with NRC (Canada), and NSC and ASIAA (Taiwan), in cooperation with the Republic of Chile. The Joint ALMA Observatory is operated by ESO, AUI/NRAO, and NAOJ. The NRAO is a facility of the National Science Foundation operated under cooperative agreement by Associated Universities, Inc. Based on observations made with the NASA/ESA Hubble Space Telescope, obtained from the data archive at the Space 
Telescope Science Institute (STScI). Some of the HST data presented in this paper were obtained from the Mikulski Archive for Space Telescopes (MAST), others were acquired under program HST-GO-13730 with support provided by NASA through a grant from the STScI. STScI is operated by the Association of Universities for Research in Astronomy, Inc., under NASA contract NAS5-26555. The Mopra radio telescope is part of the Australia Telescope National Facility, which is funded by the Australian Government for operation as a National Facility managed by CSIRO. Based on observations carried out under project number 209-14 with the IRAM $30 \mathrm{~m}$ Telescope. IRAM is supported by INSU/CNRS (France), MPG (Germany), and IGN (Spain).

\section{References}

Aalto, S., Garcia-Burillo, S., Muller, S., et al. 2012, A\&A, 537, A44

Alatalo, K., Blitz, L., Young, L. M., et al. 2011, ApJ, 735, 88

Alatalo, K., Lacy, M., Lanz, L., et al. 2015, ApJ, 798, 31

Bohlin, R. C., Savage, B. D., \& Drake, J. F. 1978, ApJ, 224, 132

Bolatto, A. D., Warren, S. R., Leroy, A. K., et al. 2013, Natur, 499, 450

Booth, C. M., Agertz, O., Kravtsov, A. V., \& Gnedin, N. Y. 2013, ApJL, 777, L16

Brüggen, M., \& Scannapieco, E. 2016, ApJ, 822, 31

Bustard, C., Zweibel, E. G., \& D'Onghia, E. 2016, ApJ, 819, 29

Cardelli, J. A., Clayton, G. C., \& Mathis, J. S. 1989, ApJ, 345, 245

Cecil, G., Bland-Hawthorn, J., Veilleux, S., \& Filippenko, A. V. 2001, ApJ, 555,338

Cooper, J. L., Bicknell, G. V., Sutherland, R. S., \& Bland-Hawthorn, J. 2009, ApJ, 703, 330

Dickinson, A. S., Phillips, T. G., Goldsmith, P. F., Percival, I. C., \& Richards, D. 1977, A\&A, 54, 645

Engelbracht, C. W., Kundurthy, P., Gordon, K. D., et al. 2006, ApJL, 642, L127

Faucher-Giguère, C.-A., \& Quataert, E. 2012, MNRAS, 425, 605

Faure, A., Varambhia, H. N., Stoecklin, T., \& Tennyson, J. 2007, MNRAS, 382,840

Flower, D. R., Pineau Des Forêts, G., \& Walmsley, C. M. 2007, A\&A, 474, 923

Fujita, A., Martin, C. L., Mac Low, M.-M., New, K. C. B., \& Weaver, R. 2009, ApJ, 698, 693

Gao, Y., \& Solomon, P. M. 2004, ApJS, 152, 63

García-Burillo, S., Combes, F., Usero, A., et al. 2014, A\&A, 567, A125

Girichidis, P., Naab, T., Walch, S., et al. 2016, ApJL, 816, L19

Heckman, T. M., Lehnert, M. D., Strickland, D. K., \& Armus, L. 2000, ApJS, 129,493

Heesen, V., Beck, R., Krause, M., \& Dettmar, R.-J. 2009, A\&A, 494, 563

Hlavacek-Larrondo, J., Carignan, C., Daigle, O., et al. 2011, MNRAS, 411, 71

Kim, J.-G., Kim, W.-T., \& Ostriker, E. C. 2016, ApJ, 819, 137

Knudsen, K. K., Walter, F., Weiss, A., et al. 2007, ApJ, 666, 156

Leroy, A. K., Bolatto, A. D., Ostriker, E. C., et al. 2015b, ApJ, 801, 25

Leroy, A. K., Walter, F., Martini, P., et al. 2015a, ApJ, 814, 83
Lindberg, J. E., Aalto, S., Muller, S., et al. 2016, A\&A, 587, A15

Lovell, A. J., Kallivayalil, N., Schloerb, F. P., et al. 2004, ApJ, 613, 615

Marcolini, A., Strickland, D. K., D'Ercole, A., Heckman, T. M., \& Hoopes, C. G. 2005, MNRAS, 362, 626

McCourt, M., O'Leary, R. M., Madigan, A.-M., \& Quataert, E. 2015, MNRAS, 449,2

McCourt, M., Oh, S. P., OLeary, R. M., \& Madigan, A.-M. 2016, arXiv:1610. 01164

Meier, D. S., \& Turner, J. L. 2012, ApJ, 755, 104

Meier, D. S., Walter, F., Bolatto, A. D., et al. 2015, ApJ, 801, 63

Müller-Sánchez, F., González-Martín, O., Fernández-Ontiveros, J. A., Acosta-Pulido, J. A., \& Prieto, M. A. 2010, ApJ, 716, 1166

Murray, N., Ménard, B., \& Thompson, T. A. 2011, ApJ, 735, 66

Nayakshin, S., \& Zubovas, K. 2012, MNRAS, 427, 372

Osterbrock, D. E., \& Ferland, G. J. 2006, Astrophysics of Gaseous Nebulae and Active Galactic Nuclei (2nd ed.; Sausalito, CA: Univ. Science Books)

Rekola, R., Richer, M. G., McCall, M. L., et al. 2005, MNRAS, 361, 330

Roussel, H., Wilson, C. D., Vigroux, L., et al. 2010, A\&A, 518, L66

Rupke, D. S., Veilleux, S., \& Sanders, D. B. 2002, ApJ, 570, 588

Rupke, D. S. N., \& Veilleux, S. 2013, ApJL, 775, L15

Saha, S., Ray, S., Bhattacharyya, B., \& Barua, A. K. 1981, PhRvA, 23, 2926

Sakamoto, K., Ho, P. T. P., Iono, D., et al. 2006, ApJ, 636, 685

Salas, P., Galaz, G., Salter, D., et al. 2014, ApJ, 797, 134

Salem, M., \& Bryan, G. L. 2014, MNRAS, 437, 3312

Scannapieco, E., \& Brüggen, M. 2015, ApJ, 805, 158

Sharp, R. G., \& Bland-Hawthorn, J. 2010, ApJ, 711, 818

Shirley, Y. L. 2015, PASP, 127, 299

Simpson, C. M., Pakmor, R., Marinacci, F., et al. 2016, ApJL, 827, L29

Skatrud, D. D., De Lucia, F. C., Blake, G. A., \& Sastry, K. V. L. N. 1983, JMoSp, 99, 35

Stacey, G. J. 2011, ITTST, 1, 241

Strickland, D. K., Heckman, T. M., Weaver, K. A., \& Dahlem, M. 2000, AJ, 120,2965

Strickland, D. K., Heckman, T. M., Weaver, K. A., Hoopes, C. G., \& Dahlem, M. 2002, ApJ, 568, 689

Sturm, E., González-Alfonso, E., Veilleux, S., et al. 2011, ApJL, 733, L16

Thompson, T. A., Fabian, A. C., Quataert, E., \& Murray, N. 2015, MNRAS, 449, 147

Thompson, T. A., Quataert, E., Zhang, D., \& Weinberg, D. H. 2016, MNRAS, 455,1830

Turner, B. E. 1985, ApJ, 299, 312

Uhlig, M., Pfrommer, C., Sharma, M., et al. 2012, MNRAS, 423, 2374

Ulvestad, J. S., \& Antonucci, R. R. J. 1997, ApJ, 488, 621

Usero, A., Leroy, A. K., Walter, F., et al. 2015, AJ, 150, 115

Veilleux, S., Cecil, G., Bland-Hawthorn, J., et al. 1994, ApJ, 433, 48

Veilleux, S., Cecil, G., \& Bland-Hawthorn, J. 2005, ARA\&A, 43, 769

Veilleux, S., Meléndez, M., Sturm, E., et al. 2013, ApJ, 776, 27

Veilleux, S., Rupke, D. S. N., \& Swaters, R. 2009, ApJL, 700, L149

Walter, F., Weiss, A., \& Scoville, N. 2002, ApJL, 580, L21

Watson, A. M., Gallagher, J. S., III, Holtzman, J. A., et al. 1996, AJ, 112, 534

Westmoquette, M. S., Smith, L. J., \& Gallagher, J. S., III 2011, MNRAS, 414, 3719

Zhang, D., \& Davis, S. W. 2016, arXiv:1612.00022

Zubovas, K., \& King, A. 2012, ApJL, 745, L34 